\title{
Ground Displacements due to the Deformations of Shallow Tunnels with Arbitrary Cross Sections in Soft Ground
}

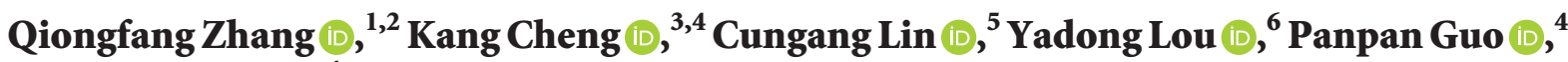 \\ and Xiaolu Gan (iD) \\ ${ }^{1}$ Power China Huadong Engineering Corporation Limited, Hangzhou 311122, Zhejiang, China \\ ${ }^{2}$ Engineering Research Center of Smart Rail Transportation of Zhejiang Province, Hangzhou 310014, Zhejiang, China \\ ${ }^{3}$ China Railway 11th Bureau Group Co., Ltd., Wuhan 430061, China \\ ${ }^{4}$ Research Center of Coastal and Urban Geotechnical Engineering, Zhejiang University, Hangzhou 310058, China \\ ${ }^{5}$ School of Civil Engineering, Sun Yat-Sen University, Guangzhou 510275, China \\ ${ }^{6}$ Hangzhou Metro Group Co., Ltd., Hangzhou 310003, Zhejiang, China \\ Correspondence should be addressed to Cungang Lin; lincg@mail.sysu.edu.cn
}

Received 5 March 2021; Accepted 25 June 2021; Published 8 July 2021

Academic Editor: Hang Chen

Copyright (c) 2021 Qiongfang Zhang et al. This is an open access article distributed under the Creative Commons Attribution License, which permits unrestricted use, distribution, and reproduction in any medium, provided the original work is properly cited.

\begin{abstract}
Nowadays, a huge number of shield-driven tunnels with noncircular cross sections are constructed in urban areas all around the world. However, the ground displacements associated with tunneling still form a difficult issue, especially for noncircular tunnels. In this study, an analytical solution is derived to estimate the ground displacements induced by the deformations of shallow noncircular tunnels in soft ground. First, a solution for the stresses and displacements around a deep tunnel in a full plane is formulated by imposing a specified convergence pattern over the cavity boundary. Subsequently, this solution is validated using finite element simulations in a case study of an elliptical tunnel with four different convergence patterns. Afterward, the solution in the full plane is extended to a half plane using the virtual image technique to estimate the ground displacements around shallow tunnels. The solution is also validated using finite element simulations.
\end{abstract}

\section{Introduction}

In the last twenty years, a variety of special-shaped shields have been developed to build tunnels with many different shapes and cross sections [1-7]. Compared with the most widely used circular tunnels, noncircular tunnels are believed to have the advantage of making full use of excavated space [8]. Thus, they have been more and more frequently employed in urban areas for transportation development.

During the construction of shallow tunnels in soft ground, one critical concern is the ground movements associated with tunneling. At present, there have been several methods available for predicting tunneling-induced ground movements, including empirical methods, analytical solutions, model tests, and numerical simulations [9]. Although numerical simulations are generally powerful and elaborate, analytical solutions are still necessary, as they are less timeconsuming and easier for practical applications compared with other methods $[6,10]$. In particular, when abundant experiences are obtained from field observations and model tests, analytical solutions with empirical modifications can provide as many accurate results as numerical simulations.

Among analytical solutions, those derived from elastic theories make up important categories. These elastic solutions can generally be formulated by a combination of complex variable methods [11] and some well-established theorems, such as the Cauchy's integral theorem [12], Laurent's theorem [13], and theorems on conformal mapping [12]. The elastic solutions for generated stresses and displacements around deep tunnels in rocks are mostly derived in infinite planes by imposing a specified loading distribution over boundaries at certain distances from 
tunnels [14-17]. However, since the tectonic stresses acting on shallow openings are not as high as those acting on deep openings, such pressure-controlled derivation is generally not applicable to shallow tunnels [15]. Instead, a displacement-controlled method, in which, for example, a specified convergence pattern is imposed on the cavity of a shallow tunnel and is employed for estimating the soil stresses and displacements around shallow tunnels [18-24]. Sagaseta [22] regarded the excavation of a tunnel as the radial convergence of a small circle to the tunnel axis. The authors of [23] developed the study of [22] by considering the effect of Poisson's ratio of soil and an elliptical convergence pattern around a tunnel boundary. The authors of $[19,20]$ derived elastic solutions for soil displacements around both deep and shallow tunnels in clay by assuming four different convergence patterns. Pinto and Whittle [21] pointed out that tunneling-induced ground settlements depend on convergence patterns that are both uniformly radial and elliptical. Overall, it has been proven by previous studies that the ground displacements due to the excavations of shallow circular tunnels can be reliably calculated under appropriately assumed convergence patterns around cavities.

More and more tunnels with noncircular cross sections are constructed worldwide [25-29]. However, most of the abovementioned solutions for tunneling-induced soil stresses and displacements are related to circular tunnels, giving sparse attention to noncircular tunnels. Wang et al. [30] derived an elastic solution for shallow tunnels with arbitrary cross sections in rock and stiff soil using the Schwartz alternating method [31]. It is obtained by superimposing an infinite plane solution [32] and a half-plane solution [33]. However, this solution is only applicable to deep tunnels.

In this study, an elastic solution is derived to estimate the ground displacements around shallow tunnels in soft ground. First of all, an elastic solution in a full plane is formulated to calculate the ground displacements around deep tunnels with arbitrary cross sections. Afterward, it is employed in the deep tunnels of several commonly used shapes. Furthermore, the solution is extended to a half-plane for shallow tunnels using the virtual image technique [22].

\section{Elastic Solutions in a Full Plane}

2.1. Formulations. In this section, the issue to be solved is the soil stresses and displacements around a deep tunnel with an arbitrary cross section in an infinite plane. It is assumed that the cavity occupied by the tunnel is vacuum and that the surrounding soil is homogeneous, isotropic, and linearly elastic. Elastic solutions for soil stresses and displacements can be obtained by following the steps as follows:

(1) Conformally mapping the glyphs in the original domain onto the regular graphs in the mapped plane

(2) Translating the conditions of the displacement boundary or stress boundary into a complex variable domain using the conformal mapping technique
(3) Getting the unknown parameters of the stress function while assuming that the stress of a point at infinity is zero

(4) Using the available theorems, such as Cauchy's integral theorem and Laurent's theorem, to simplify the stress function

2.1.1. Complex Variable Function. In the absence of body forces, the stresses around a cavity can be solved using the Airy stress function $F$ as

$$
\frac{\partial^{4} F}{\partial y^{4}}+2\left(\frac{\partial^{4} F}{\partial x^{2} \partial y^{2}}+\frac{\partial^{4} F}{\partial x^{4}}\right)=0,
$$

where $x$ and $y$ denote the horizontal and vertical axes, respectively, in a Cartesian coordinate system, as shown in Figure 1.

The equilibrium can be satisfied if $F$ meets the following conditions [12]:

$$
\begin{gathered}
\sigma_{x}=\frac{\partial^{2} F}{\partial y^{2}}, \\
\sigma_{y}=\frac{\partial^{2} F}{\partial x^{2}}, \\
\tau_{x y}=-\frac{\partial^{2} F}{\partial x \partial y},
\end{gathered}
$$

where $\sigma_{x}$ and $\sigma_{y}$ are the normal stresses in the $x$ and $y$ directions, respectively, and $\tau_{x y}$ is the shear stress in the $x-y$ plane.

The displacements around the cavity can be expressed by the two harmonic functions, $\varphi(z)$ and $\psi(z)$, as follows [34]:

$$
2 G\left(u_{x}+i u_{y}\right)=\kappa \varphi(z)-z \overline{\varphi^{\prime}(z)}-\overline{\psi(z)},
$$

where $u_{x}$ and $u_{y}$ are the displacements in the $x$ and $y$ directions, respectively; $G$ is the shear modulus of the soil; $z$ is a complex variable in the $z$-plane, $z=x+i y$; the constant $\kappa$ is related to Poisson's ratio $v$ by $\kappa=3-4 v$ for the plane strain problems and $\kappa=3-v / 1+v$ for the plane stress problems, respectively; $\nu$ is Poisson's ratio of soil; and $\varphi(z)$ and $\psi(z)$ are functions with respect to the variable $z$, which can be determined by giving the displacement boundary conditions around the tunnel cavity. $\overline{\varphi^{\prime}(z)}$ and $\overline{\psi(z)}$ are the conjugates of $\overline{\varphi^{\prime}(z)}$ and $\overline{\psi(z)}$, respectively.

Afterward, the stresses around the cavity can be expressed in the form of complex functions as follows:

$$
\left\{\begin{array}{l}
\sigma_{x}+\sigma_{y}=4 \operatorname{Re}\left[\varphi^{\prime}(z)\right], \\
\left(\sigma_{y}-\sigma_{x}\right)+2 i \tau_{x y}=2\left[\bar{z} \varphi^{\prime \prime}(z)+\psi^{\prime}(z)\right],
\end{array}\right.
$$

where $\bar{z}$ is the conjugate of $z$ and $\operatorname{Re}[$.$] denotes the real part$ of a generic complex variable $[\cdot]$. 


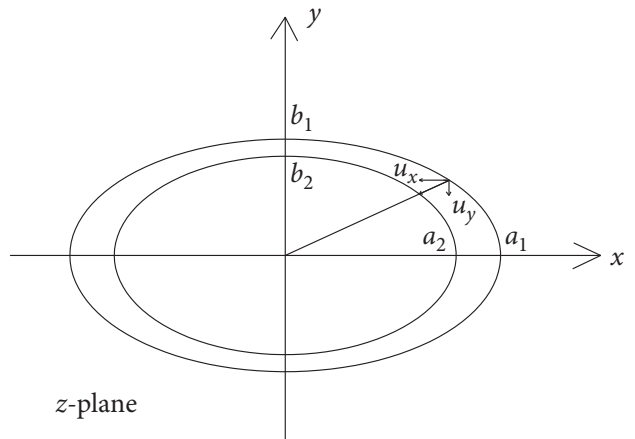

Figure 1: The $z$-plane. transformed into a unit circular hole in the $\zeta$-plane, as depicted in Figure 2. The employment of the mapping technique $z=w(\zeta)$ yield

$$
\left\{\begin{array}{l}
\varphi(z)=\varphi(w(\zeta))=\varphi(\zeta), \\
\psi(z)=\psi(w(\zeta))=\psi(\zeta) .
\end{array}\right.
$$

For a 2-dimensional (2D) elastic problem, the displacements and stresses are expressed in terms of two complex variable functions, $\varphi(z)$ and $\psi(z)$, with $z=x+i y$ and $i=\sqrt{-1}$. By introducing conformal mapping and equation (5), the generated stresses and displacements around the tunnel cavity can be expressed as follows:

2.1.2. Conformal Mapping. A tunnel with an arbitrary cross section (ellipse, circle, oval, and rectangle) in the $z$-plane is

$$
\begin{aligned}
& 2 G\left(u_{x}+i u_{y}\right)=\kappa \varphi(\zeta)-\frac{w(\zeta)}{\overline{w^{\prime}(\zeta)}} \overline{\varphi^{\prime}(\zeta)}-\overline{\psi(\zeta)}, \\
& \left\{\begin{array}{l}
\sigma_{x}+\sigma_{y}=4 \operatorname{Re}\left[\frac{\varphi^{\prime}(\zeta)}{w^{\prime}(\zeta)}\right], \\
\left(\sigma_{y}-\sigma_{x}\right)+2 i \tau_{x y}=\frac{2}{w^{\prime}(\zeta)}\left[\overline{w(\zeta)}\left(\frac{\varphi^{\prime}(\zeta)}{w^{\prime}(\zeta)}\right)^{\prime}+\psi^{\prime}(\zeta)\right],
\end{array}\right.
\end{aligned}
$$

where $\overline{w(\zeta)}$ is the conjugate of the complex function $w(\zeta), \zeta$ is a complex variable in the $\zeta$-plane, $\zeta=\xi+\mathrm{i} \eta=\rho e^{\mathrm{i} \vartheta}, \xi$ and $\eta$ are variables of the Cartesian coordinates system in the mapped plane, and $\rho$ and $\vartheta$ are variables of the polar coordinates system in the mapped plane.

From (7), the stress components can be obtained as follows:

$$
\left\{\begin{array}{l}
\tau_{x y}=\operatorname{Im}\left[\frac{1}{w^{\prime}(\zeta)}\left(\overline{w(\zeta)}\left(\frac{\varphi^{\prime}(\zeta)}{w^{\prime}(\zeta)}\right) \prime+\psi^{\prime}(\zeta)\right)\right] \\
\sigma_{x}=2 \operatorname{Re}\left[\frac{\varphi^{\prime}(\zeta)}{w^{\prime}(\zeta)}\right]-\operatorname{Re}\left[\frac{1}{w^{\prime}(\zeta)}\left(\overline{w(\zeta)}\left(\frac{\varphi^{\prime}(\zeta)}{w^{\prime}(\zeta)}\right) \prime+\psi^{\prime}(\zeta)\right)\right] \\
\sigma_{x}=2 \operatorname{Re}\left[\frac{\varphi^{\prime}(\zeta)}{w^{\prime}(\zeta)}\right]-\operatorname{Re}\left[\frac{1}{w^{\prime}(\zeta)}\left(\overline{w(\zeta)}\left(\frac{\varphi^{\prime}(\zeta)}{w^{\prime}(\zeta)}\right) \prime+\psi^{\prime}(\zeta)\right)\right]
\end{array}\right.
$$

where $\operatorname{Im}[\cdot]$ denotes the imaginary component of the generic complex variable $[\cdot]$.

Since (6) is analytic in the mapped $\zeta$-plane, it can be expanded in the Laurent series of $\sigma$ using $\zeta=\rho e^{\mathrm{i} \vartheta}=\rho \sigma$ as follows:

$$
2 G\left(u_{x}+i u_{y}\right)=\sum_{-\infty}^{\infty} A_{k} e^{i n \theta}=\sum_{-\infty}^{\infty} A_{n} \sigma^{n}=g(\sigma),
$$

where $\sigma$ denotes a point on the unit circle in the $\zeta$-plane. Therefore, the coefficients $A_{k}$ are determined by means of 


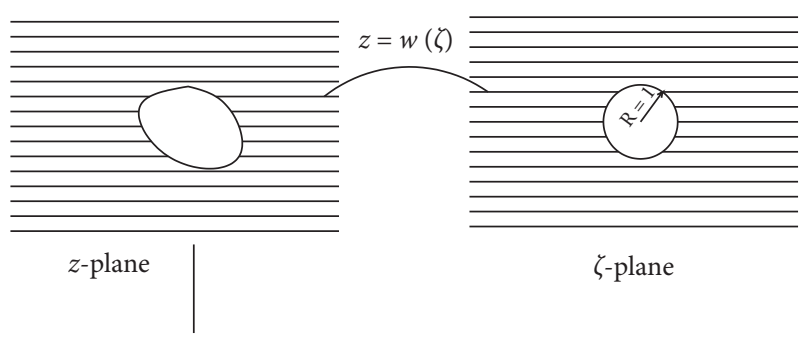

FIgURE 2: Conformal mapping of the exterior of an unlined tunnel of any shape onto the exterior of a unit circle.

the recursive relations derived from the given boundary conditions.

The displacement components can be obtained as follows:

$$
\left\{\begin{array}{l}
u_{x}=\operatorname{Re}[g(\sigma)]=\frac{1}{2 G} \operatorname{Re}\left[\sum_{-\infty}^{\infty} A_{n} \sigma^{n}\right], \\
u_{y}=\operatorname{Im}[g(\sigma)]=\frac{1}{2 G} \operatorname{Im}\left[\sum_{-\infty}^{\infty} A_{n} \sigma^{n}\right] .
\end{array}\right.
$$

As shown by Muskhelishvili, the stress boundary condition in the phase plane can be expressed as follows:

$$
\varphi(\zeta)+\frac{w(\zeta)}{\overline{w(\zeta)}} \overline{\varphi^{\prime}(\zeta)}+\overline{\psi(\zeta)}=i \int\left(f_{x}+f_{y}\right) \mathrm{d} s,
$$

where $f x$ and $f y$ are the surface stresses in the $x$ and $y$ directions, respectively, where they act on the elliptical cavity wall.

It can be verified [34] that the potentials can be written in the form as follows:

$$
\begin{aligned}
& \varphi(\zeta)=\frac{1}{8 \pi(1-v)}\left(\overline{F_{x}}+i \overline{F_{y}}\right) \ln \zeta+B w(\zeta)+\varphi_{0}(\zeta) \\
& \psi(\zeta)=-\left(\frac{3-4 v}{8 \pi(1-v)}\right)\left(\overline{F_{x}}-i \overline{F_{y}}\right) \ln \zeta+(B \prime+i C \prime) w(\zeta)+\varphi_{0}(\zeta)
\end{aligned}
$$

where $\overline{F_{x}}+i \overline{F_{y}}$ is the resultant force applied to the boundary (which in this case is equal to zero). The variables $B, B+i C$, and $B^{\prime}-i C^{\prime}$ can be written as

$$
\begin{aligned}
B & =\frac{\left(\sigma_{1}+\sigma_{2}\right)}{4}, \\
B^{\prime}+i C^{\prime} & =-\left(\frac{1}{2}\right)\left(\sigma_{1}-\sigma_{2}\right) e^{-2 i \alpha},
\end{aligned}
$$

where $\sigma_{1}$ and $\sigma_{2}$ are the principal stresses at infinity and $\alpha$ is the angle between the maximum principal direction and $x$ direction.

It is assumed that the stresses vanished at infinity and on the tunnel boundary. Thus,

$$
\begin{aligned}
\overline{F_{x}}+i \overline{F_{y}} & =0, \\
B^{\prime}+i C^{\prime} & =0 .
\end{aligned}
$$

By substituting (16) into (12) and (13),

$$
\left\{\begin{array}{l}
\varphi(\zeta)=\varphi_{0}(\zeta) \\
\psi(\zeta)=\psi_{0}(\zeta)
\end{array}\right.
$$

where the functions $\varphi_{0}(\zeta)$ and $\psi_{0}(\zeta)$ are single-valued and analytic in the $\zeta$-plane, including the point at infinity.

According to equations (6), (9), and (17),

$$
g(\sigma)=\sum_{-\infty}^{\infty} A_{n} \sigma^{n}=(3-4 v) \varphi_{0}(\sigma)-\left(\frac{w(\sigma)}{\overline{w^{\prime}(\sigma)}}\right) \overline{\varphi_{0}^{\prime}(\sigma)}-\overline{\psi_{0}(\sigma)}
$$

By taking the conjugate, then

$$
\overline{g(\sigma)}=\sum_{-\infty}^{\infty} \overline{A_{n} \sigma^{-n}}=(3-4 v) \overline{\varphi_{0}(\sigma)}-\frac{\overline{w(\sigma)}}{w^{\prime}(\sigma)} \varphi_{0}^{\prime}(\sigma)-\psi_{0}(\sigma) .
$$

Equations 19 and 20 are multiplied by $d \sigma / 2 \pi i(\sigma-\zeta)$ and integrated along the unit circle in the $\zeta_{\text {-plane: }}$

$$
\begin{aligned}
& \frac{1}{2 \pi i}(3-4 v) \oint \frac{\varphi_{0}(\sigma)}{\sigma-\zeta} d \sigma-\frac{1}{2 \pi i} \oint \frac{w(\sigma)}{\overline{w^{\prime}(\sigma)}} \frac{\overline{\varphi_{0}^{\prime}(\sigma)}}{\sigma-\zeta} d \sigma-\frac{1}{2 \pi i} \oint \frac{\overline{\psi_{0}(\sigma)}}{\sigma-\zeta} d \sigma=\frac{1}{2 \pi i} \oint \frac{g(\sigma)}{\sigma-\zeta} d \sigma, \\
& \frac{1}{2 \pi i}(3-4 v) \oint \frac{\overline{\varphi_{0}(\sigma)}}{\sigma-\zeta} d \sigma-\left(\frac{1}{2 \pi i} \oint \frac{\overline{w(\sigma)}}{w^{\prime}(\sigma)} \frac{\varphi_{0}^{\prime}(\sigma)}{\sigma-\zeta} d \sigma-\frac{1}{2 \pi i} \oint \frac{\psi_{0}(\sigma)}{\sigma-\zeta} d \sigma\right)=\frac{1}{2 \pi i} \oint \frac{\overline{g(\sigma)}}{\sigma-\zeta} d \sigma . \\
& \text { the properties of the Cauchy integral, } \\
& \varphi_{0}(\zeta)=-\left(\frac{1}{3-4 v}\right) \frac{1}{2 \pi i} \oint \frac{g(\sigma)}{\sigma-\zeta} d \sigma .
\end{aligned}
$$
$\overline{w(\sigma)}, w^{\prime}(\sigma)$, and $\varphi_{0}^{\prime}(\sigma)$ are analytic functions in the unit circle. Then, $\overline{w(\sigma)} / w^{\prime}(\sigma) \varphi_{0}^{\prime}(\sigma)$ is analytic in the unit circle. It can be concluded that the second and third terms on the lefthand side of (17) are zero and that the first term on the lefthand side of (18) is zero. Thus, it can be concluded that
By combining (9) and (23),

$$
\varphi_{0}(\zeta)=\left(\frac{1}{3-4 v}\right) \sum_{-\infty}^{0} A_{n} \zeta^{n}
$$


A similar process could be performed to find $\psi_{0}(\zeta)$, where $A_{n}$ are the only unknown constants in the two complex variable functions.

2.1.3. Tunnels with Different Cross Sections. To find a solution for the present problem, we first consider the transformation of the tunnels with arbitrary cross sections, such as ellipses, circles, oval shapes, and squares, in the $z$ plane into a unit circular hole in the $\zeta$-plane. The transformation function [35] is assumed as

$$
\begin{aligned}
& z=w(\varsigma)=R\left(\varsigma+\sum_{n=1}^{N} a_{n} \varsigma^{-n}\right), \\
& \varsigma=\rho e_{i \theta},
\end{aligned}
$$

where $R$ is a real number that refers to the cross-section size and $a_{n}$ is a general complex coefficient satisfying $\left|a_{n}<1 / n\right|$ [36]. Inverse mapping is analytical, single-valued, and nonzero in the exterior part of the curve.

In many instances, it is assumed that the physical domain possesses $p$ symmetry axes and then yields

$$
z=w(\varsigma)=R\left(\varsigma+\sum_{n=1}^{N} a_{1 p n} \varsigma^{1-p n}\right) .
$$

The tunnel cavity assigns the prescribed radial displacement along its boundary, and the central position of the curve remains unchanged after deformation. It remains axisymmetric along the $x$-axis and $y$-axis. After deformation, the curve can be expressed as

$$
Z=w_{c}(\zeta)=R_{c}\left(\zeta+\sum_{n=1}^{N} b_{n} \zeta^{-n}\right)
$$

The total displacement $u_{k}$ at the tunnel boundary can be expressed as

$$
\begin{aligned}
u & =Z-z, \\
& =\left(u_{x}+i u_{y}\right), \\
& =\left(R_{c}-R\right) \zeta+\sum_{n=1}^{N}\left(R_{c} b_{n}-R a_{n}\right) \zeta^{-n} .
\end{aligned}
$$
follows:

Compared with (10), the coefficient $A_{k}$ is obtained as

$$
\left\{\begin{array}{l}
A_{1}=2 G\left(R_{c}-R\right) \\
A_{n}=0 \quad n \geq 2 \\
A_{n}=2 G\left(R_{c} b_{k}-R a_{k}\right) \quad n \leq-1 .
\end{array}\right.
$$

For a cylindrical cavity of radius $r$ in isotropic soil, the boundary condition is solved by mapping onto a circle of a unit radius:

$$
z=r \zeta
$$

When undergoing uniform convergence $u_{\varepsilon}$, the deformed tunnel boundary is expressed as follows:

$$
Z=\left(r-u_{\varepsilon}\right) \zeta
$$

Considering an infinite plane containing an elliptical tunnel cavity with a major axis $2 a_{1}$ and a minor axis $2 b_{1}$, the transformation function became

$$
\begin{aligned}
z & =w(\zeta), \\
& =R_{0}\left(\zeta+m \zeta^{-1}\right),
\end{aligned}
$$

where $R_{0}=\left(a_{1}+b_{1}\right) / 2$ and $m=\left(a_{1}-b_{1}\right) /\left(a_{1}+b_{1}\right)$.

Heller et al. [37] provide a mapping function for a rectangular opening of unit width and height, $\mathrm{K}$, using the Schwarz-Christoffel integral:

$$
z=R\left[\zeta+\left(\frac{1-K^{2}}{4} \frac{1}{\zeta}\right)-\left(\frac{K^{2}}{24} \frac{1}{\zeta^{3}}\right)-\left(\frac{K^{2}\left(1-K^{2}\right)}{160} \frac{1}{\zeta^{5}}\right)-\left(\frac{K^{2}\left(1-3 K^{2}+K^{4}\right)}{896} \frac{1}{\zeta^{7}}\right)+\cdots\right]
$$

Manh et al. [16] propose that, to get a smaller error for the elastic fields at the corner of a rectangular opening, at least 10 terms must be used in the conformal mapping functions. Once an approximate polynomial mapping for the origin and deformed shape of a cavity boundary is known, the elastic solution can be determined using the analytical method proposed in the paper. Generally, tunnel contours comprise complex curves. To generalize these studies, it is reasonable to simplify complicated tunnels as unlined tunnels with elliptical outlines [38].
2.1.4. Different Deformation Patterns. It is of practical significance to determine the coefficients of the approximate polynomial mapping function after deformation. Taking the elliptical tunnel as an example, four convergence patterns in Figure 3 are assumed to describe the displacement on the boundary of the elliptical tunnel:

$$
\begin{aligned}
& \text { B.C. }-1: u_{c}=-u_{0}\left(\sin \theta+t \cos ^{2} \theta\right), \\
& \text { B.C. }-2: u_{c}=-u_{0}\left(1+\sin \theta-(1-t) \cos ^{2} \theta\right),
\end{aligned}
$$




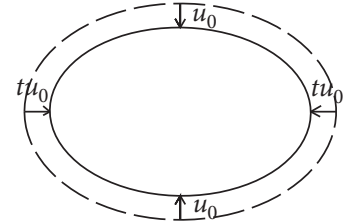

Boundary condition 1

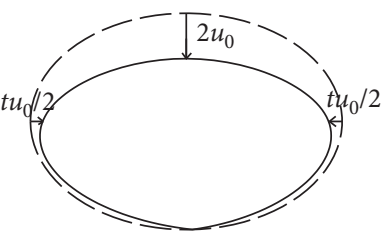

Boundary condition 3

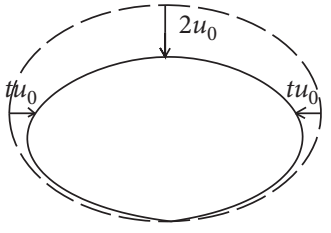

Boundary condition 2

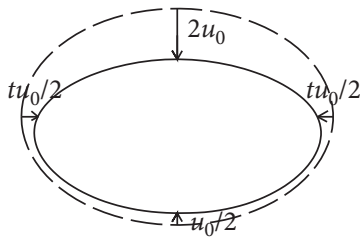

Boundary condition 4
Figure 3: Boundary conditions of the given displacement.

$$
\begin{aligned}
\text { B.C. }-3: u_{c} & =-u_{0}\left(1+\sin \theta-\left(1-\frac{t}{2}\right) \cos ^{2} \theta\right), \\
\text { B.C. }-4: u_{c} & =-\frac{u_{0}}{4}\left(5+3 \sin \theta-(5-2 t) \cos ^{2} \theta\right), \\
1 & \leq t \leq \frac{a_{1}}{b_{1}} .
\end{aligned}
$$

It should be noted that the given convergence patterns are reduced to the boundary conditions given by [19] when $t=1$ and $a_{1}=b_{1}$.

On the boundary of the elliptical cavity in the $z$-plane,

$z_{c}=x_{c}+i y_{c}=(a \cos \theta+i b \sin \theta)$
$\bar{z}_{c}=x_{c}-i y_{c}=(a \cos \theta-i b \sin \theta)$$\Rightarrow\left\{\begin{array}{l}\cos \theta=\left(\frac{z_{c}+\overline{z_{c}}}{2 a}\right), \\ \sin \theta=\left(\frac{z_{c}-\overline{z_{c}}}{2 b i}\right) .\end{array}\right.$

On the boundary of the unit circle in the $\zeta$-plane,

$$
\begin{aligned}
& \zeta=e^{i \vartheta}=\sigma, \\
& \bar{\zeta}=e^{-i \vartheta}=\sigma^{-1}, \\
& z_{c}=R\left(\zeta_{c}+\frac{m}{\zeta_{c}}\right)=R\left(\sigma+m \sigma^{-1}\right), \\
& \overline{z_{c}}=R\left(\bar{\zeta}_{c}+\frac{m}{\bar{\zeta}}\right)=R\left(\sigma^{-1}+m \sigma\right) .
\end{aligned}
$$

By substituting (35) into (3),

$$
\left\{\begin{array}{l}
\cos \theta=\frac{R(1+m)\left(\sigma+\sigma^{-1}\right)}{2 a}, \\
\sin \theta=\frac{R(1-m)\left(\sigma-\sigma^{-1}\right)}{2 b i},
\end{array}\right.
$$

$$
F(x, y)=2 G\left(u_{x_{2}}^{0}+i u_{y_{2}}^{0}\right)=2 G u_{c}(\cos \theta+i \cos \theta) .
$$

By substituting (31)-(34) and (38) into (39), the displacement can be expressed as a polynomial of $\sigma^{k}$. The constant $A_{k}$ is obtained through a comparison with (9). By substituting $A_{k}$ in (8), the stress components are found.

2.2. Application to an Elliptical Tunnel in a Full Plane. Considering that an infinite plane contains an elliptical tunnel cavity with a major axis $2 a_{1}$ and a minor axis $2 b_{1}$, as illustrated in Figure 1, a uniform radial displacement (B.C.1, $t=1)$ is assumed as the boundary condition for the elliptical tunnel cavity. The initial elliptical tunnel cavity is converged without altering the ratio of the semimajor axis to the semiminor axis of the elliptical tunnel cavity.

$$
\begin{aligned}
\frac{a_{1}}{a_{2}} & =\frac{b_{1}}{b_{2}}, \\
& =\frac{\rho_{1}}{\rho_{2}}, \\
& =\frac{1}{k},
\end{aligned}
$$

where $a_{2}$ and $b_{2}$ correspond to the current semimajor and semiminor axes of the elliptical tunnel cavity, respectively; $\rho_{1}, \rho_{2}$ denote the angular length of the elliptical tunnel at any point before and after the deformation in the polar coordinate system, respectively; and $k$ is the convergence ratio.

At the boundary of the elliptical tunnel cavity,

$$
\begin{aligned}
& u_{x}^{0}=-(1-k), \\
& x=-\frac{(1-k)(z+\bar{z})}{2}=-(1-k) R \frac{\left[(m+1) \sigma+(m+1) \sigma^{-1}\right]}{2}, \\
& u_{y}^{0}=-(1-k), \\
& y=-\frac{(1-k)(z-\bar{z})}{2}=-(1-k) R \frac{\left[(1-m) \sigma-(1-m) \sigma^{-1}\right]}{2} .
\end{aligned}
$$

By comparing (23) and (25), there are only two Fourier coefficients for the complex variable function: 


$$
\begin{aligned}
A_{1} & =2 G(1-k) R, \\
A_{-1} & =2 G(1-k) R m, \\
A_{k} & =0(k>1, k<-1) .
\end{aligned}
$$

2.2.1. Comparison of the Solutions for the Elliptical and Circular Tunnels. The ratio of the semimajor and semiminor axes of the initial elliptical cavity $(a / b)$ is $5 / 4$, and the soil Poisson's ratio equals 0.5 . The elastic solution of the elliptical tunnel cavity and the circumscribed circular tunnel cavity expansion shown in Figure 4 are obtained using the analytical method. The orthoradial displacement and shearing stress caused by the radial displacement of the circumscribed circular tunnel cavity and elliptical tunnel cavity along the semimajor and semiminor axis directions of the elliptical tunnel cavity are zero.

Figure 5 shows the radial displacement variation of the soil in relation to the distance from the center of the cavities, normalized by the radius of the circumscribed circular tunnel cavity. The analytical results of the radial displacement along the major and minor axes of the elliptical tunnel cavity are compared with the results of the circumscribed circular tunnel cavity.

It can be seen that the radial displacement of the soil around the elliptical tunnel cavity along the major and minor axes and circumscribed circular tunnel cavity decreases with the increase in the distance from the cavity's center and that it eventually tends to zero. The radial displacement of the soil along the major axis of the elliptical tunnel cavity is equal to that of the circumscribed circular tunnel cavity.

Figure 6 shows the radial and orthoradial stresses of the soil, which are caused by the radial displacement of the elliptical tunnel cavity and circumscribed circular tunnel cavity in relation to the distance from the center of the cavities. The magnitude of the radial stress of the soil along the semimajor axis of the elliptical tunnel cavity is larger than the radial stress of the soil along the radial direction of the circumscribed circular. The radial stresses along the semiminor axis of the elliptical tunnel cavity are minimum. The magnitude of the soil orthoradial stress induced by the radial displacement of the circumscribed circular tunnel cavity is larger than the soil orthoradial stress caused by the elliptical tunnel cavity. As the distance from the cavity center increased, the soil radial and orthoradial stresses around the elliptical tunnel cavity and circumscribed circular tunnel cavity tend to zero.

When $a=4 \mathrm{~m}, G=2000 \mathrm{kPa}$, and $k=0.92$ are considered, the magnitudes of the soil stress and displacement around the cavities are very small $\left(\left|\sigma_{\rho}\right|<1 \mathrm{kPa}\right.$, $\left.\left|\sigma_{\theta}\right|<1 \mathrm{kPa},\left|\tau_{\rho \theta}\right|<1 \mathrm{kPa},\left|u_{\rho}\right|<0.010 \mathrm{~mm}\right)$, at $\sim 50 \mathrm{~m}$ from the cavity center. That is, the influence radius of the convergence of the elliptical cavity is $\sim 20$ times that of the semimajor axis.
2.2.2. Elastic Solutions of the Soil around an Elliptical Tunnel Cavity Using the Analytical and Finite Element Method Methods. To validate the accuracy of the results, the solution is compared with a finite element method (FEM) calculation using the ABAQUS 2D software. The FEM model size is $1000 \times 1000 \mathrm{~m}$, and the boundary elements were infinite elements. Since the whole model is symmetric about the origin point and the $x, y$ axes, one-quarter of the whole model is selected for comparison. The soil's stress and displacement around the elliptical ellipse are compared and analyzed. Figures 7 and 8 show the contour of the normalized stresses and displacements of the soil from the analytical solution with the FEM solution. The selected parameters of the elliptical tunnel cavity and soil are as follows:

$$
\begin{aligned}
\frac{a_{1}}{H} & =\frac{5}{47}, \\
k & =0.92, \\
G & =2000 \mathrm{kPa}, \\
v & =0.4 .
\end{aligned}
$$

where $a$ is the semimajor axis, $k$ is the convergence ratio, $G$ is the shear modulus, and $v$ is the soil Poisson's ratio.

It can be seen from Figures $7(\mathrm{a})-7(\mathrm{e})$ that the soil shearing stress in the directions of the $x$-axis and the $y$-axis is zero. There are some differences between the values of the vertical stresses and displacements because the boundary condition of the ABAQUS model is finite and fixed at the bottom. The soil stress and displacement calculated using the complex function method and FEM are approximately equal, which verifies the correctness of the theoretical method in this paper.

\subsubsection{Elastic Solutions of the Soil around the Elliptical Tunnel} Cavity with Different Ellipticity Values. Figures 8(a)-8(e) show the soil stress and displacement caused by the convergence of the elliptical cavities with different ellipticity values. From the figures, it can be seen that the ellipticity of the cavity decreased, the soil stress in the $x$-axis direction of the soil increased, and that the soil stress and displacement in the $y$-axis direction decreases. Also, the soil displacement in the $x$-axis direction can be seen.

\section{Elastic Solutions in a Half-Plane}

3.1. Virtual Image Technique. So far, the displacement fields are considered in an infinite plane. The ground displacement induced by the deformation of the tunnel cavity with the arbitrary cross section in the half-plane can be obtained using the virtual image technique.

Figure 9 shows the diagram of the virtual image technique used in this paper. Supposing that $y=0$ is the surface, there are two convergence elliptical cavities at the points $O_{1}$ $(0,-h)$ and $\mathrm{O}_{2}(0, h)$. Then, at the surface, 


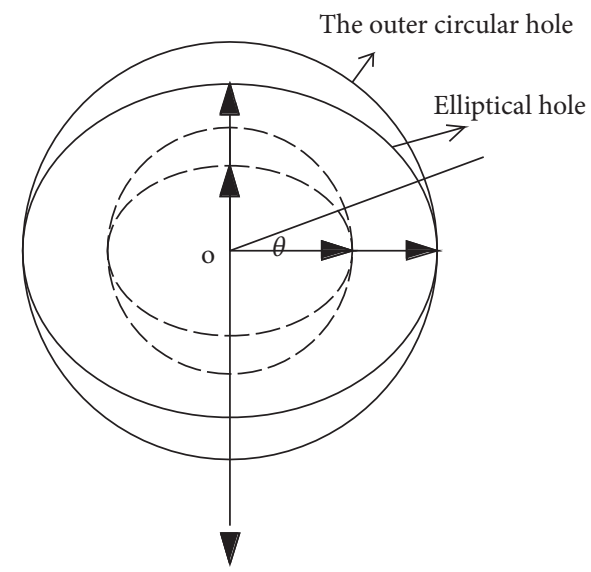

Figure 4: Current elliptical tunnel cavity and circumscribed circular tunnel cavity before and after the radial displacement at the boundary of the original tunnel cavity.

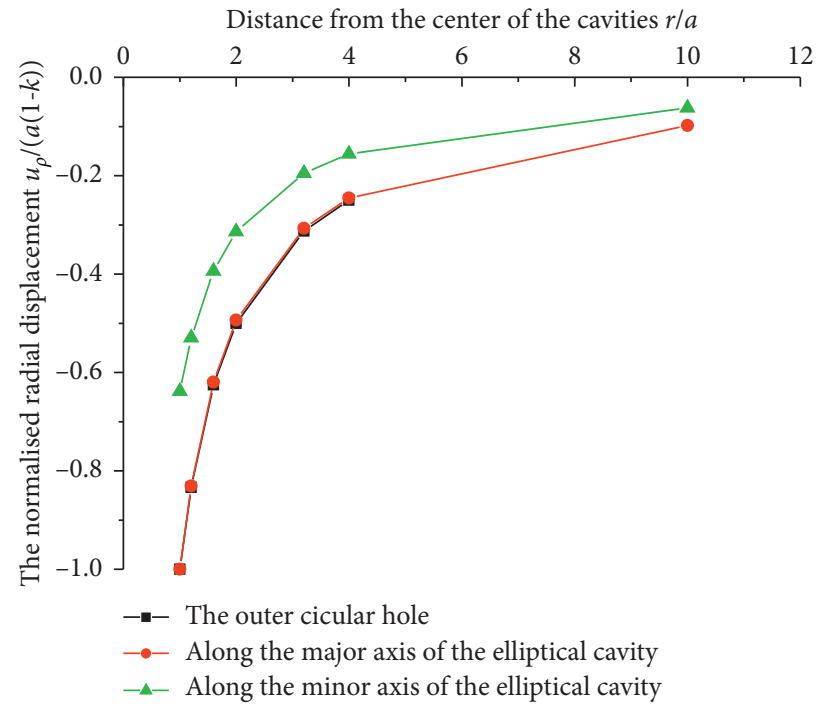

Figure 5: Distribution of the radial displacement of the soil due to the radial displacement at the boundaries of the cavities.

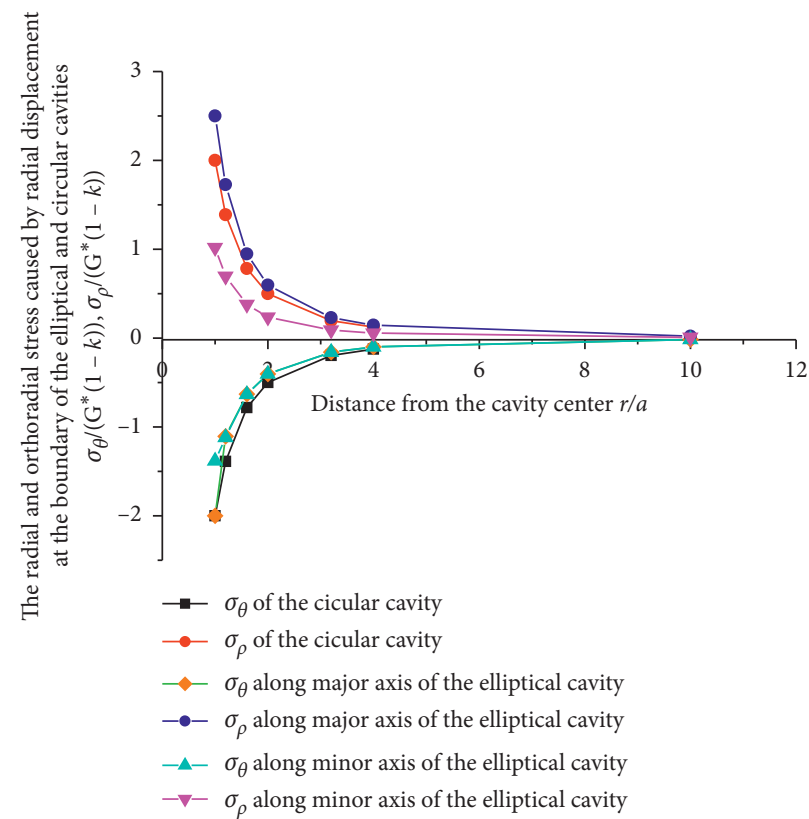

Figure 6: Distribution of the stress of the soil elements due to the radial displacement at the boundaries of the cavities. 


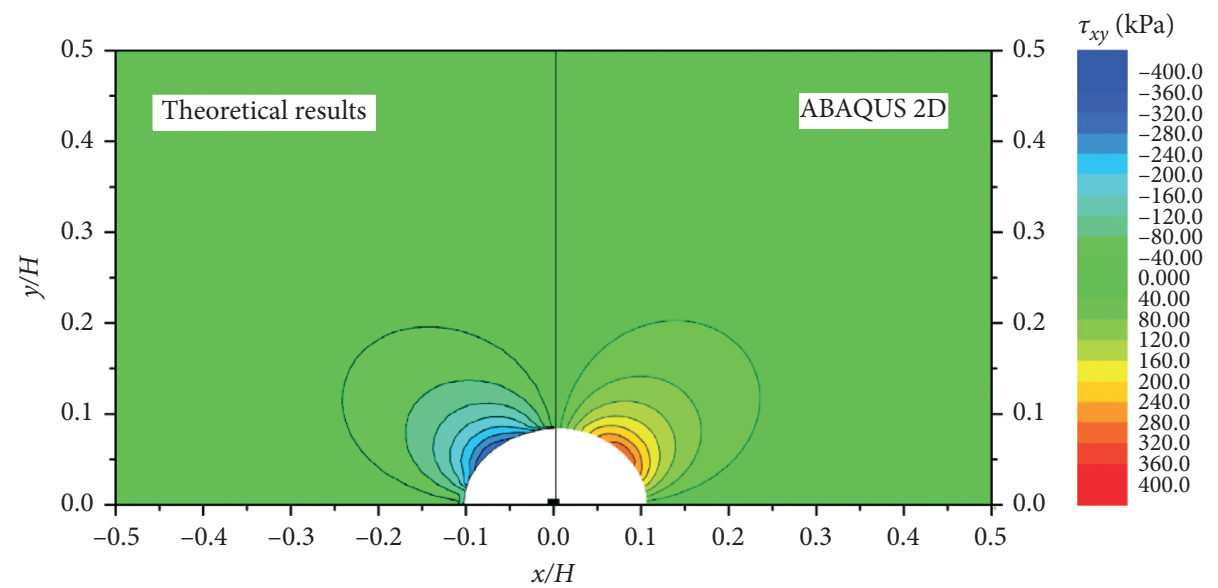

(a)

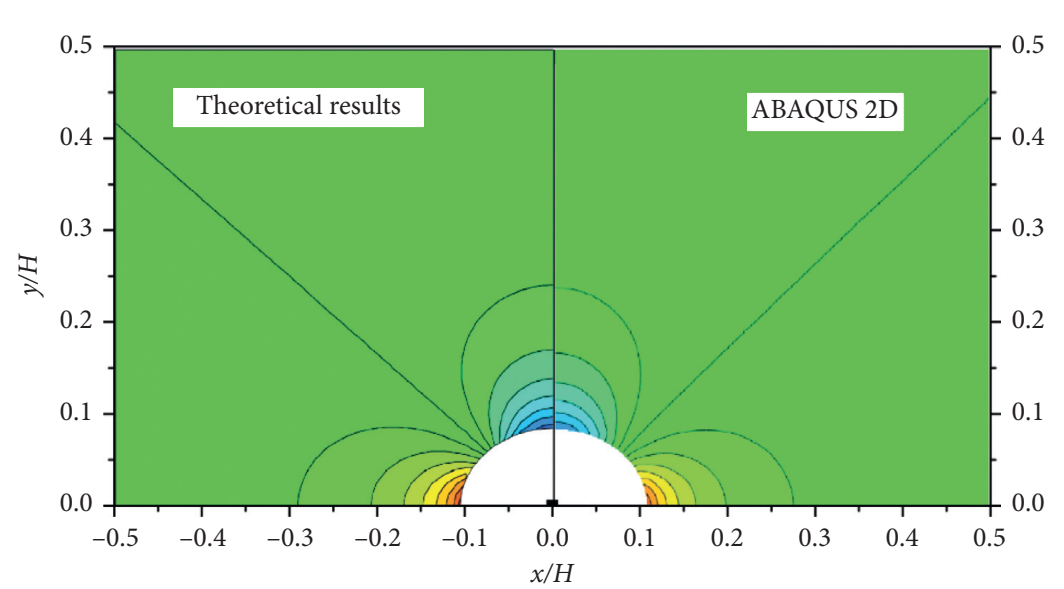

$\sigma_{x}(\mathrm{kPa})$

(b)

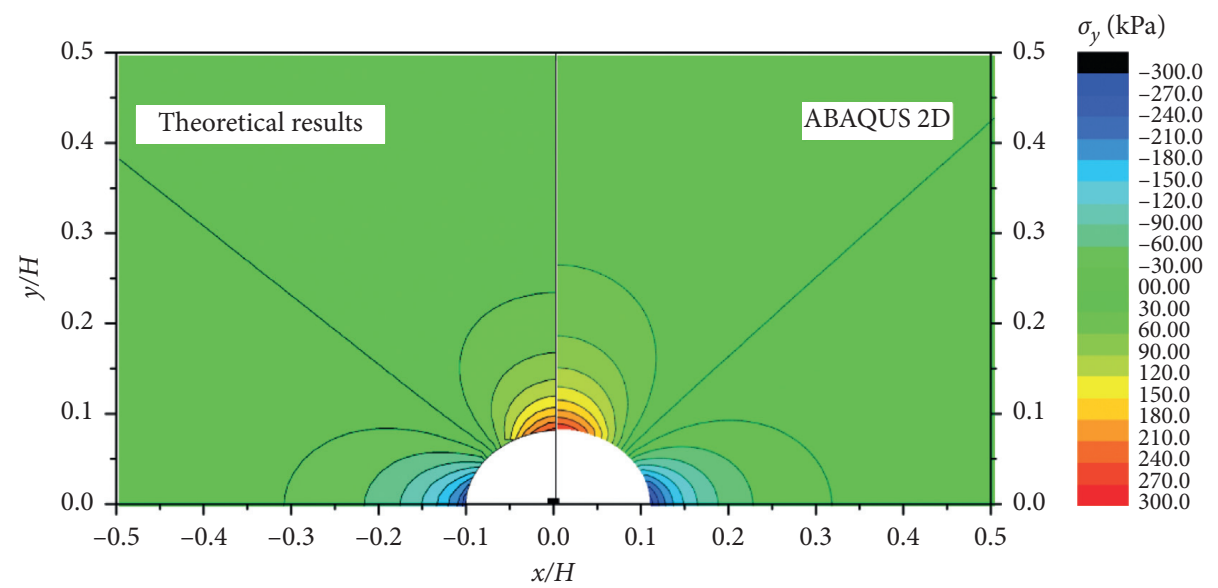

(c)

Figure 7: Continued. 


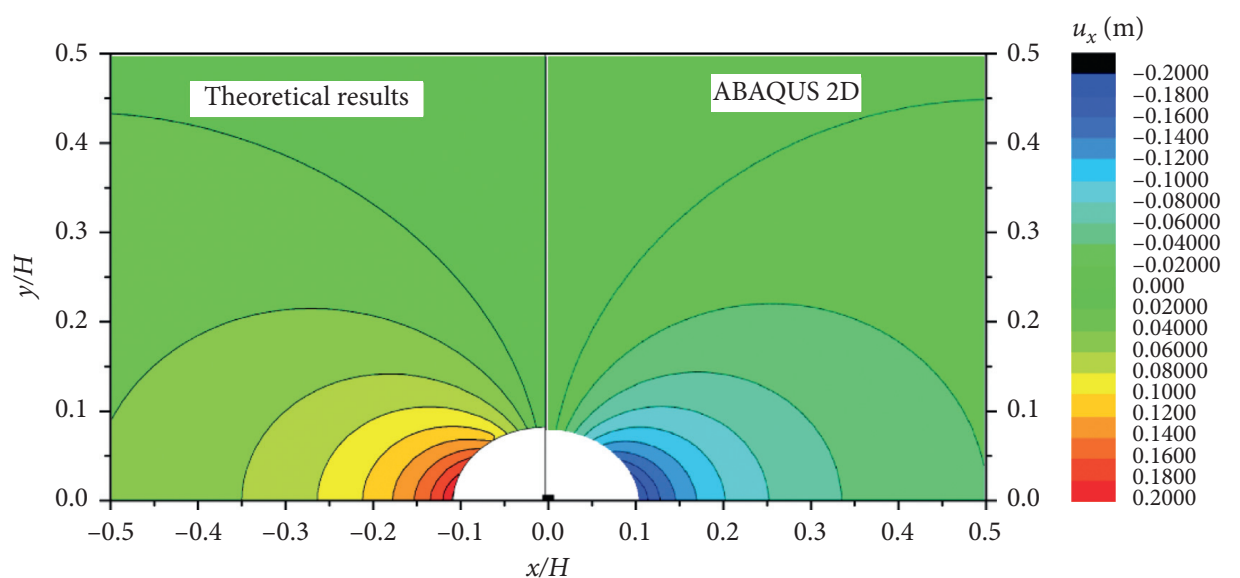

(d)

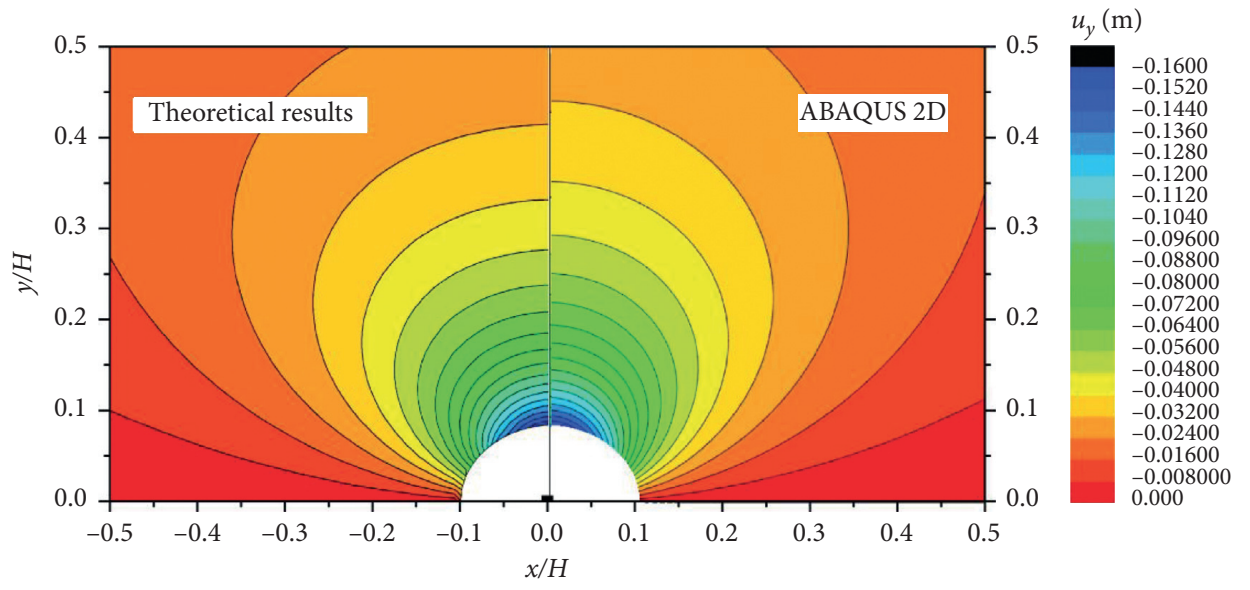

(e)

Figure 7: Contours of the normalized stresses and displacements in the case of $(a) /(b)=2$ as derived by the theoretical method and ABAQUS 2D. (a) Shear stress of the soil. (b) Soil stress in the $x$-direction. (c) Soil stress in the $y$-direction. (d) Soil displacement in the $x$ direction. (e) Soil displacement in the $y$-direction.

$$
\begin{aligned}
\left.u_{y}\right|_{y=0} & =0 ; \\
\left.u_{x}\right|_{y=0} & =2 u_{x}(x, h), \\
\left.\sigma_{y}\right|_{y=0} & =2 \sigma_{y}(x, h) ; \\
\left.\tau_{x y}\right|_{y=0} & =0 .
\end{aligned}
$$

The boundary of the half-plane is considered to be stressfree. The normal stresses at the surface $y=0$ are made to be equal to zero, meaning that it is necessary to apply a reverse force to balance the normal stress at the surface induced by the two convergence elliptical tunnel cavities. This problem turns into a Boussinesq problem for the half plane $y \leq 0$ with the following boundary conditions:

$$
\begin{aligned}
y & =0: \sigma_{y 1}=-2 \sigma_{y}(x, 0), \\
\tau_{x y}(x, 0) & =0 .
\end{aligned}
$$

In the case of a circular tunnel, the ground displacement induced by the reverse vertical stress applied to the ground surface can be solved using the Fourier formula [23]. When the section shape of the tunnel is elliptical, the vertical stress expression is complex, and the Fourier transform is no longer applicable. In this paper, the numerical solution of the surface displacement can be derived using the formula proposed by [39].

Reference [39] uses the complex variable function and virtual image technique to obtain the displacement caused 


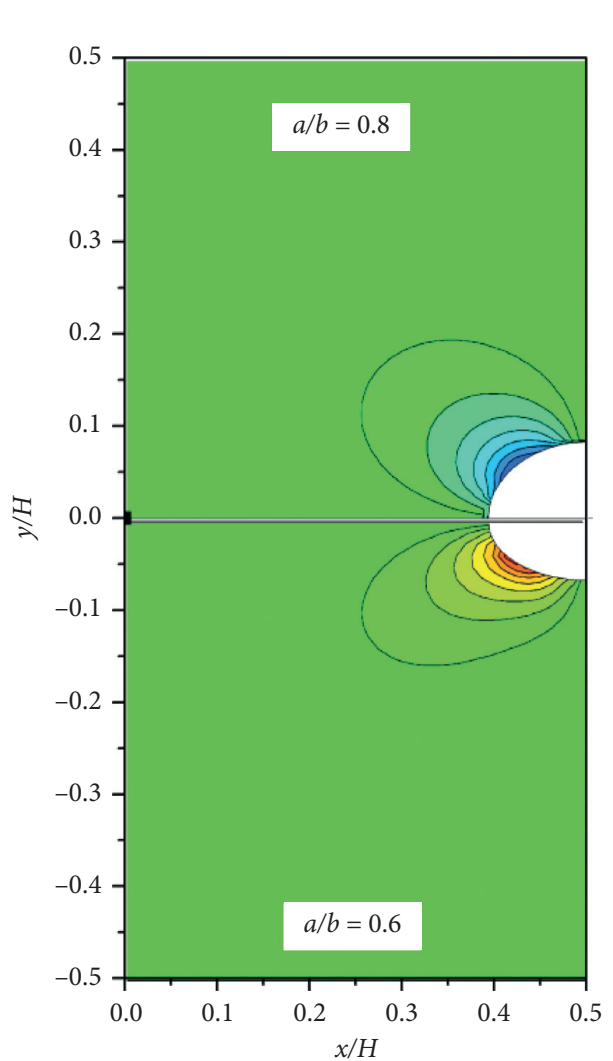

(a)

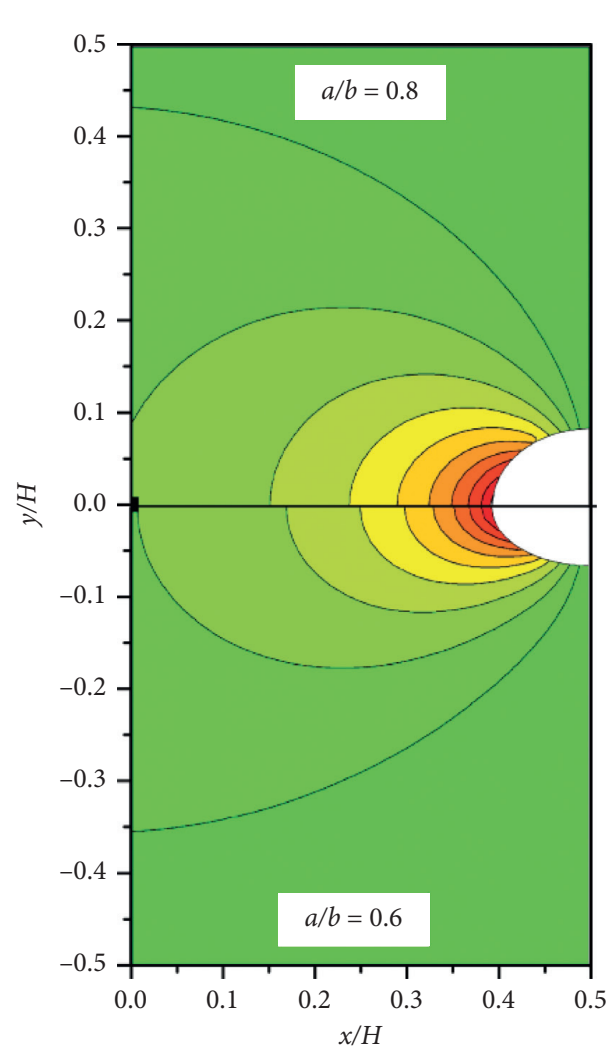

(c)

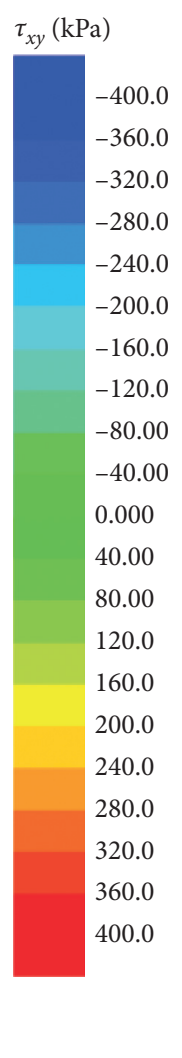

$u_{x}(\mathrm{~m})$
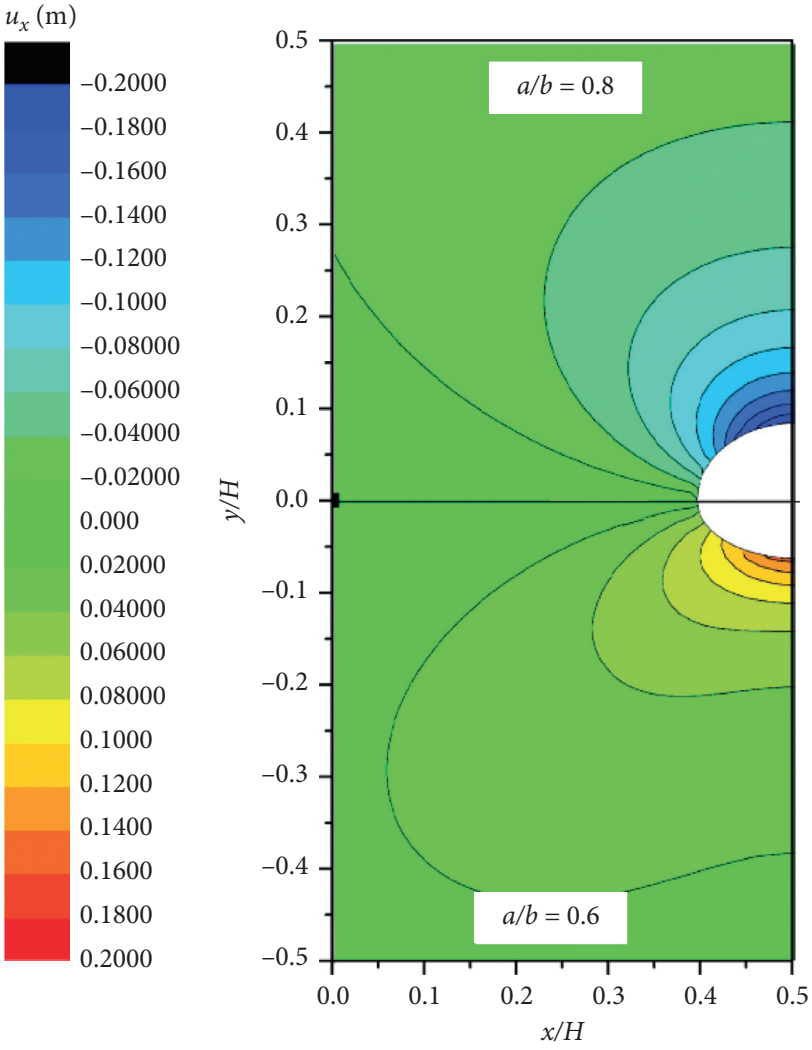

$u_{y}(\mathrm{~m})$

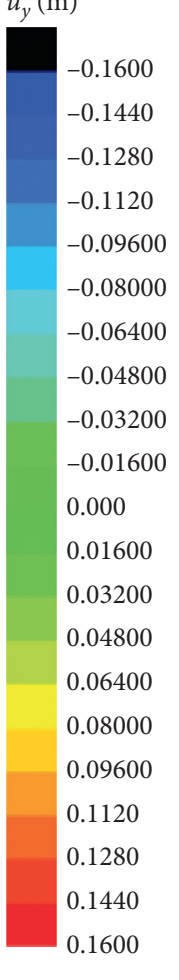

$\sigma_{x}(\mathrm{kPa})$
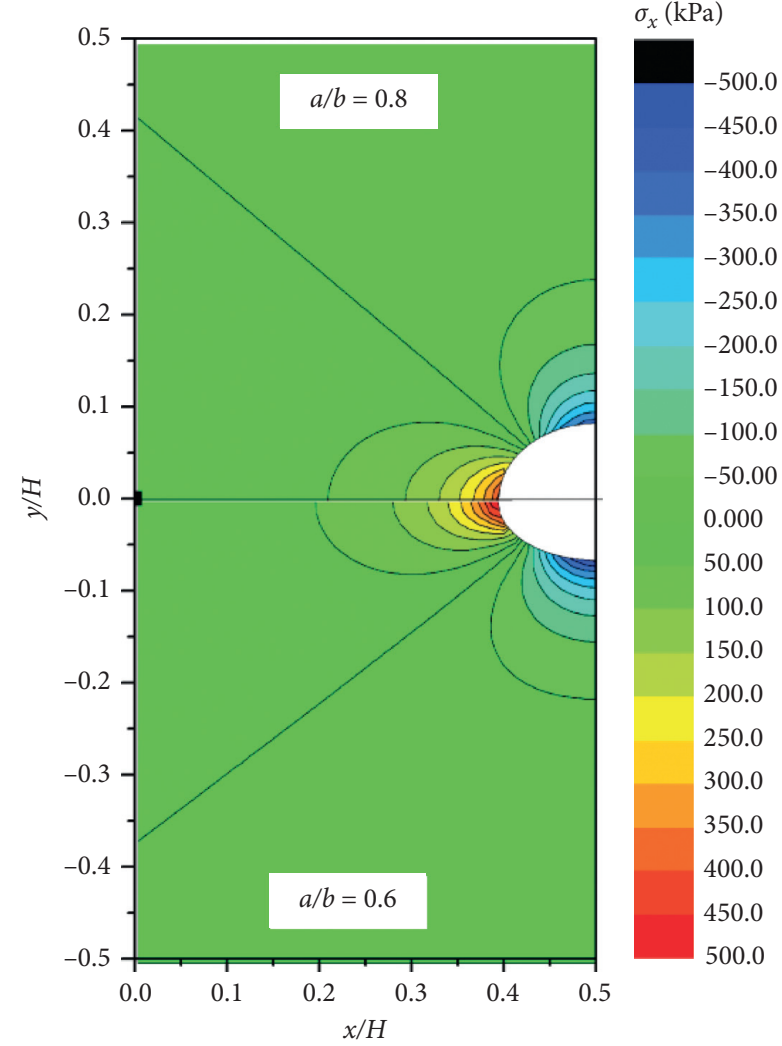

$-450.0$

$-400.0$

$-350.0$

$-300.0$

$-250.0$

$-200.0$

$-150.0$

$-100.0$

$-50.00$

0.000

50.00

100.0

150.0

200.0

250.0

300.0

350.0

400.0

450.0

500.0

(b)

(d)

Figure 8: Continued. 


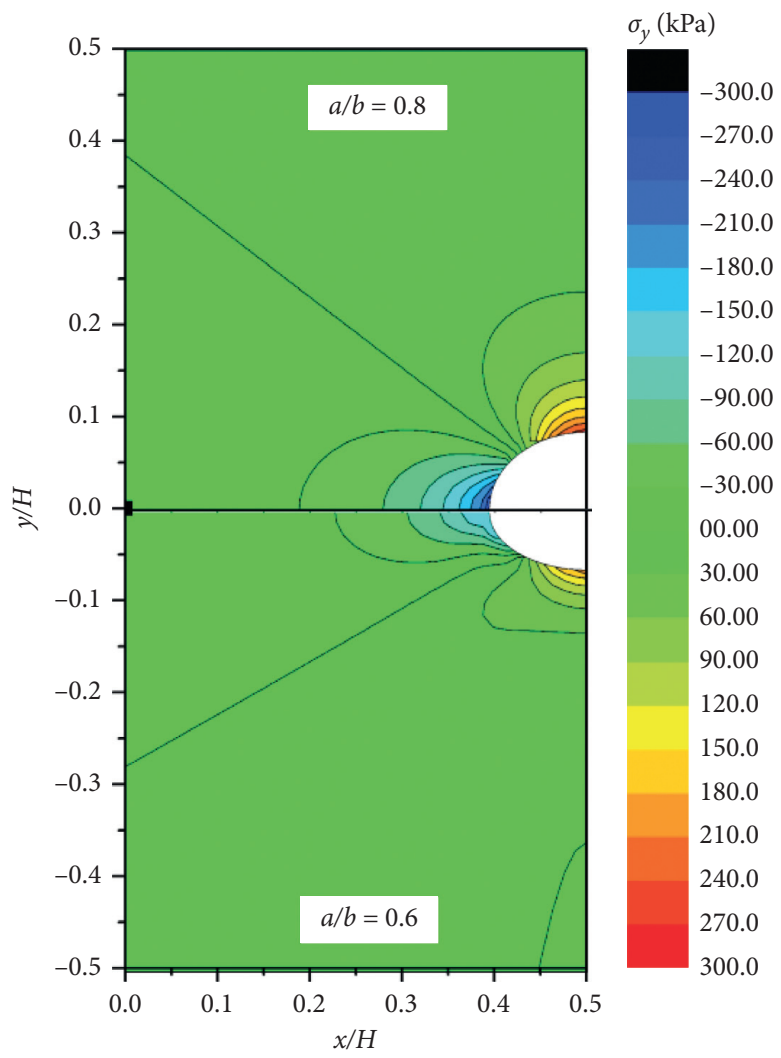

(e)

Figure 8: Contours of the normalized stresses and displacements in the case of the elliptical tunnel with different ellipticity values. (a) Shearing stress of soil. (b) Soil stress in the $x$-direction. (c) Soil displacement in the $x$-direction. (d) Soil displacement in the $y$-direction. (e) Soil stress in the $y$-direction.

by the vertical and horizontal concentrated forces at any point in the half plane. When a unit vertical concentrated force applies to a point $m$ in the half plane, as illustrated in Figure 10, the stress function becomes

$$
\begin{aligned}
\varphi_{1}(z)= & \frac{i P}{8 \pi(1-v)} \log \left(\frac{z-i h}{z+i h}\right)-\frac{i P}{2 \pi} \log (z-i h)+\frac{P}{4 \pi(1-v)} \frac{h}{z-i h}, \\
\psi_{1}(z)= & \frac{i P}{8 \pi(1-v)}\left[(3-4 v) \log \left(\frac{z-i h}{z+i h}+\frac{i h}{z-i h}+\frac{i h}{z+i h}\right)\right], \\
& -\frac{i P}{2 \pi} \log (z-i h)-\left(\frac{P(1-2 v)}{4 \pi(1-v)} \frac{i h}{z-i h}\right)+\frac{P}{4 \pi((1-v))} \frac{h z}{(z-i h)^{2}}, \\
2 G\left(u_{x}+i u_{y}\right)= & \kappa \varphi_{1}(z)-z \overline{\varphi_{1}^{\prime}(z)}-\overline{\psi_{1}(z) .}
\end{aligned}
$$

Similarly, the stress function is obtained when a unit horizontal concentrated force is applied to a point $m$ in the half plane, as seen in Figure 10(b). Supposing that $h=0$ in (44) and (43), the surface displacement caused by the unit force applied to the surface can be obtained.

Figure 11 shows a schematic diagram of solving the vertical displacement method. Due to the concentrated force $P$ applied to the boundary, the subsidence of the point $K$ to the boundary (the distance from the origin is $r$ ) relative to the subsidence of the reference point $B$ (the distance from the origin is $s$ ) is

$$
\eta=\frac{2 P\left(1-v^{2}\right)}{\pi E} \ln \frac{s}{r}
$$

The relative horizontal displacement is 


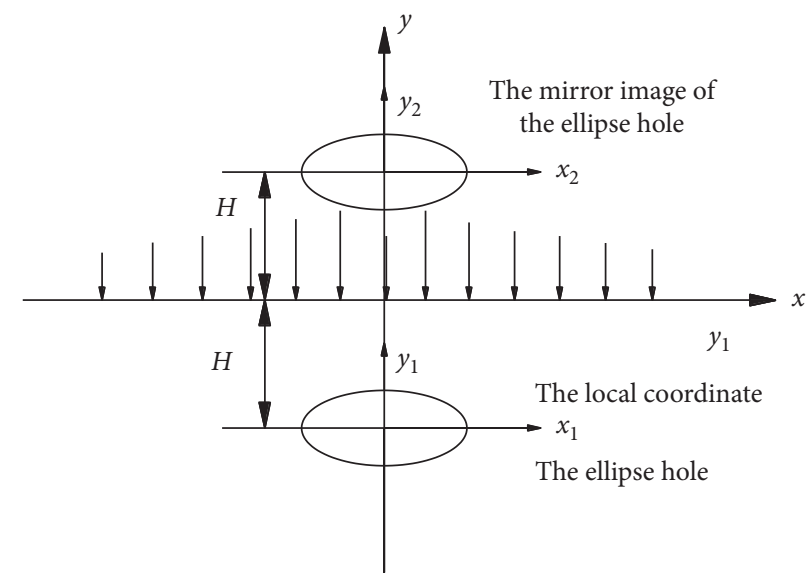

FIGURE 9: Diagram of the virtual image technique.

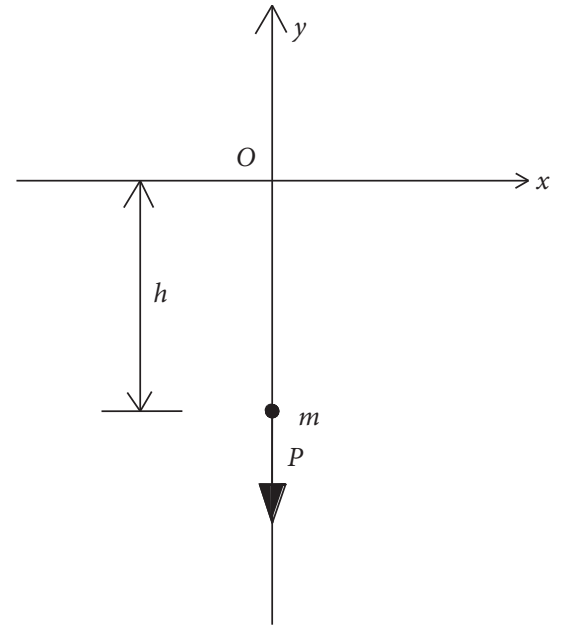

(a)

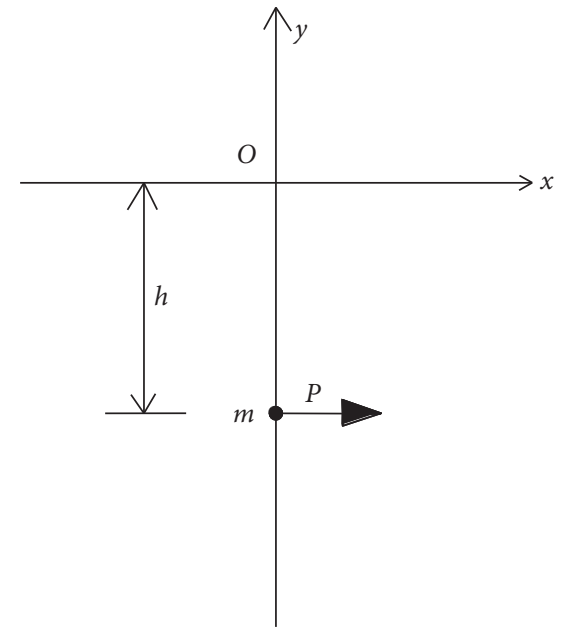

(b)

FIGURE 10: Schematic diagram of the vertical and horizontal concentrated forces applied to the half-plane.

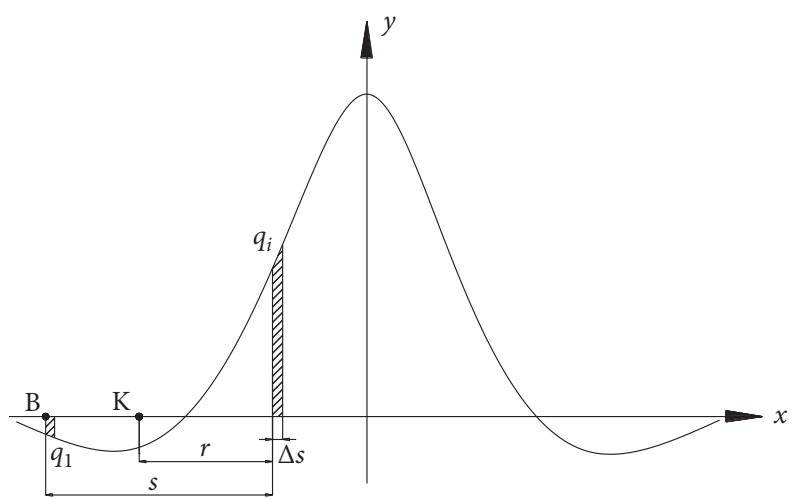

FIGURE 11: Schematic diagram of solving the vertical displacement method. 


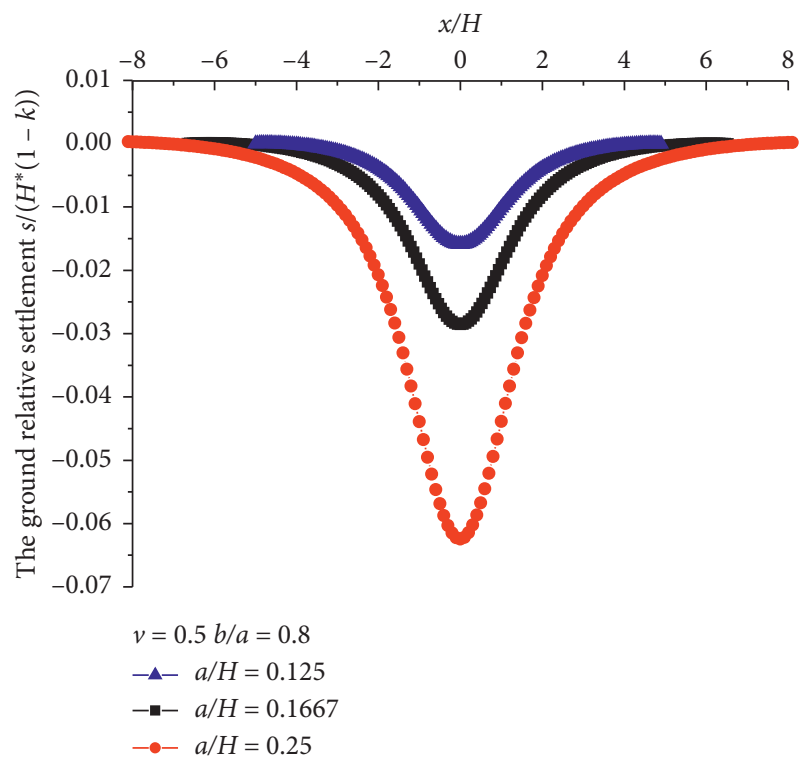

Figure 12: Vertical displacement of ground when $a / H$ changes.

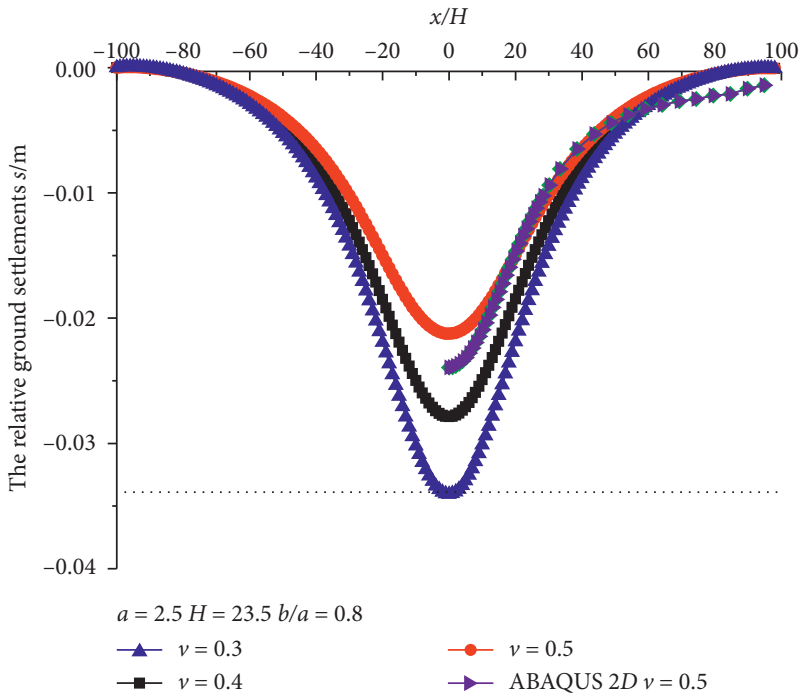

(a)

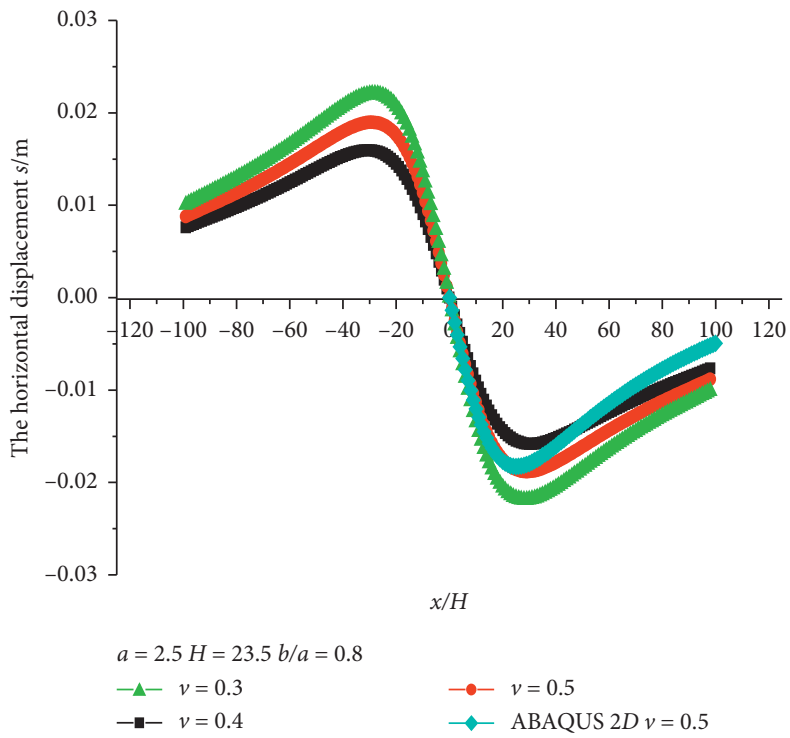

(b)

FIGURE 13: Influence of Poisson's ratio on the horizontal and vertical displacements of ground induced by the elliptical tunnel cavity's deformation. (a) Vertical displacement of ground. (b) Horizontal displacement of ground.

$$
u_{x}=-\frac{1+v}{\pi E}\left[\frac{(3-4 v)}{4(1-v)} \ln \frac{r^{2}}{s^{2}}+\left(\frac{(1-2 v)^{2}}{2(1-v)}\right) \ln \frac{r}{s}\right],
$$

where $r$ is the distance from the position of the concentrated force $P$ to the point $K$ and $s$ is the distance between the position of the concentrated force $P$ and the reference point $B$. The method adopted in this paper is mainly to divide the applied uneven vertical load into $n$ parts. As shown in Figure 10, the vertical load applied to the boundary was symmetrical about the $y$-axis, and the magnitude of the vertical load at infinity was zero. The reference point $B$ is the start point of the 1st load, and point $K$ is the start point of the $j$-th load. The settlement of point $K$ could be expressed as follows:

$$
\begin{aligned}
\eta_{K} & =\sum_{i=1}^{n} \frac{2 q_{i} \Delta s}{\pi E} \ln \frac{(i-1) \Delta s}{\left|(i-1) \Delta s-r_{B K}\right|} \quad r_{B K} \neq(i-1) \Delta s, \\
r_{B K} & =(j-1) \Delta s .
\end{aligned}
$$

The relative settlement of point $K$ to the point $B$ could be expressed as follows: 


$$
\eta_{K}=\sum_{i=1}^{n} \frac{2 q_{i} \Delta s}{\pi E} \operatorname{In} \frac{(i-1)}{|(i-j)|} \quad i \neq j .
$$

The farther the distance from the reference point $B$ to the origin point and the finer the load element division, the more accurate the results.

3.2. Vertical and Horizontal Displacements Induced by the Elliptical Tunnel. Figure 12 shows that when the buried depth of the tunnel decreased, the ground displacement caused by the elliptical tunnel decreased. The vertical displacement of any point on the ground in Figure 13(a) is relative to the vertical displacement of point $x=-100$. Since the horizontal stress applied to the ground is symmetrical about the $y$-axis, the horizontal displacement of the ground should be symmetrical with respect to the origin point, and the horizontal displacement of the origin point is zero, so the relative horizontal displacement of any point subtracts the horizontal displacement of the origin point can obtain the absolute value of the horizontal displacement of the point, as shown in Figure 13(b). It can be seen from the above two figures that when the depth of the elliptical tunnel decreased, the surface displacement caused by the contraction of the elliptical tunnel gradually decreases. When Poisson's ratio decreases, the relative vertical displacement and horizontal displacement of the ground caused by the elliptical tunnel increases.

\section{Conclusions}

The paper proposes an analytical method for modeling ground displacements for the tunnels with arbitrary cross sections in clay. The following main conclusions are drawn:

(1) Once an approximate polynomial mapping for the original and deformed shapes of a cavity boundary is obtained, the elastic solution in the full plane can be determined using the proposed analytical method in this paper.

(2) The magnitude of the radial stress of the soil along the semimajor axis of the elliptical tunnel cavity is larger than the radial stress of the soil along the radial direction of the circumscribed circular. The magnitude of the orthoradial stress of the soil induced by the radial displacement of the circumscribed circular tunnel cavity is larger than the orthoradial stresses of the soil caused by the elliptical tunnel cavity. Also, the influence radius of the convergence of the elliptical cavity is 20 times that of the semimajor axis in the full plane.

(3) A good agreement of elastic solutions is found between the analytical solutions and FEM results in the full plane for the elliptical tunnel. As the distance from the cavity center increases, the soil radial and orthoradial stresses around the elliptical tunnel cavity decrease.

(4) The ellipticity of the tunnel cavity decreases, and the soil stress in the $x$-axis direction of the soil increases.
Also, the soil displacement in the $x$-axis direction and the soil stress and displacement in the $y$-axis direction decrease.

(5) The surface displacement in the half-plane can be obtained using the virtual image technique. When the depth of the elliptical tunnel decreases, the surface displacement caused by the contraction of the elliptical tunnel gradually decreases. When Poisson's ratio of the soil decreases, the relative vertical and horizontal displacements of the ground caused by the elliptical tunnel increase.

The solution is under the assumption of elasticity and the certain deformation at the tunnel boundary, and the surcharge loadings and internal forces are not considered in the derivation. The solution is only valid for the shallow tunnels excavated in clay.

\section{Data Availability}

The data used to support the findings of this study are available from the corresponding author upon request.

\section{Conflicts of Interest}

The authors declare that there are no conflicts of interest regarding the publication of this paper.

\section{Acknowledgments}

This work was financially supported by the National Natural Science Foundation of China (grant no. 41702313), advanced programs from Doctoral Fund of Zhejiang Province, the R\&D Program RENSSKADE II/REMEDY at Norwegian Geotechnical Institute, and the China Postdoctoral Science Foundation (grant nos. 2018M630468 and 2018T110407). The authors gratefully acknowledge the support provided by Norwegian Geotechnical Institute, the Research Council of Norway, and China Scholarship Council.

\section{References}

[1] A. J. Abbo, D. W. Wilson, S. W. Sloan, and A. V. Lyamin, "Undrained stability of wide rectangular tunnels," Computers and Geotechnics, vol. 53, no. 3, pp. 46-59, 2013.

[2] B. Chow, "Double-O-Tube shield tunneling technology in the shanghai rail transit project," Tunnelling and Underground Space Technology, vol. 21, no. 6, pp. 594-601, 2006.

[3] R. Liang, C. Kang, L. Xiang et al., "Responses of in-service shield tunnel to overcrossing tunneling in soft ground," Environmental Earth Sciences, vol. 80, p. 183, 2021.

[4] H. Nakamura, T. Kubota, M. Furukawa, and T. Nakao, "Unified construction of running track tunnel and crossover tunnel for subway by rectangular shape double track crosssection shield machine," Tunnelling and Underground Space Technology, vol. 18, no. 2, pp. 253-262, 2003.

[5] J. B. Si, Y. H. Zhu, C. Ji, and S. H. Zhou, "Measurement and analysis of vertical deformation of stratum induced by quasirectangular shield tunneling in soft ground," Chinese Journal of Rock Mechanics And Engineering, vol. 36, no. 6, pp. 15511558, 2017, in Chinese. 
[6] P. Guo., X. Gong, and Y. Wang, "Displacement and force analyses of braced structure of deep excavation considering unsymmetrical surcharge effect," Computers and Geotechnics, vol. 113, Article ID 103102, 2019.

[7] D. Wu, K. Xu, P. Guo, G. Lei, K. Cheng, and X. Gong, "Ground deformation characteristics induced by mechanized shield twin tunnelling along curved alignments," Advances in Civil Engineering, vol. 2021, Article ID 6640072, 17 pages, 2021.

[8] Y. Koyama, "Present status and technology of shield tunnelling method in Japan," Tunnelling and Underground Space Technology, vol. 18, pp. 145-159, 2013.

[9] R. J. Mair, "Tunnelling and geotechnics: new horizons," Géotechnique, vol. 58, no. 9, pp. 695-736, 2008.

[10] C. G. Lin, T. D. Xia, R. Z. Liang, and S. M. Wu, "Estimation of shield tunnelling-induced ground surface settlements by virtual image technique," Chinese Journal of Geotechnical Engineering, vol. 36, no. 8, pp. 1438-1446, 2014, in Chinese.

[11] A. Verruijt, "He complex variable method," Theory of Groundwater Flow, Macmillan Education, London, UK, 1982.

[12] S. G. Lekhnitskii, P. Fern, and J. J. Brandstatter, Theory of Elasticity of an Anisotropic Elastic Body, Holden Day, San Fransisco, NW, USA, 1963.

[13] A. K. Kapoor, "Cauchy's integral formula," Principles and Problem Sessions, Cambridge University Press India Private Limited, Delhi, India, 2015.

[14] A. Bobet, "Lined circular tunnels in elastic transversely anisotropic rock at depth," Rock Mechanics and Rock Engineering, vol. 44, no. 2, pp. 149-167, 2011.

[15] G. E. Exadaktylos and M. C. Stavropoulou, "A closed-form elastic solution for stresses and displacements around tunnels," International Journal of Rock Mechanics and Mining Sciences, vol. 39, no. 7, pp. 905-916, 2002.

[16] H. T. Manh, J. Sulem, and D. Subrin, “A closed-form solution for tunnels with arbitrary cross section excavated in elastic anisotropic ground," Rock Mechanics and Rock Engineering, vol. 16, no. 3, pp. 277-288, 2015.

[17] Z. Zhang and Y. Sun, "Analytical solution for a deep tunnel with arbitrary cross section in a transversely isotropic rock mass," International Journal of Rock Mechanics and Mining Sciences, vol. 48, no. 8, pp. 1359-1363, 2011.

[18] A. Verruijt, "A complex variable solution for a deforming circular tunnel in an elastic half-plane," International Journal for Numerical and Analytical Methods in Geomechanics, vol. 21, no. 2, pp. 77-89, 1997.

[19] K. H. Park, "Elastic solution for tunneling-induced ground movements in clays," International Journal of Geomechanics, vol. 4, no. 4, pp. 310-318, 2004.

[20] K. H. Park, "Analytical solution for tunnelling-induced ground movement in clays," Tunnelling and Underground Space Technology, vol. 20, no. 3, pp. 249-261, 2005.

[21] F. Pinto and A. J. Whittle, "Ground movements due to shallow tunnels in soft ground. I: analytical solutions," Journal of Geotechnical and Geoenvironmental Engineering, vol. 140, no. 4, Article ID 04013040, 2013.

[22] C. Sagaseta, "Analysis of undrained soil deformation due to ground loss," Géotechnique, vol. 37, no. 3, pp. 301-320, 1987.

[23] A. Verruijt and J. R. Booker, "Surface settlements due to deformation of a tunnel in an elastic half-plane," Géotechnique, vol. 46, no. 4, pp. 753-756, 1996.

[24] D. M. Zymnis, A. J. Whittle, and I. Chatzigiannelis, "Effect of anisotropy in ground movements caused by tunnelling," Géotechnique, vol. 63, no. 13, pp. 1083-1102, 2013.
[25] A. Inokuma, T. Ishimura, H. Asakura et al., "The design of the primary lining for an elliptical shield tunnel," in Proceeding of the International Congress towards New Worlds in Tunnelling, Helsinki, Finland, May 1992.

[26] C. He, K. Feng, and Y. Fang, "Review and prospects on constmcting technologies of metro tunnels using shield tunnelling method," Joumal of Southwest Jiaotong University, vol. 50, no. 1, pp. 97-109, 2015, in Chinese.

[27] Y. Kashima, N. Kondo, and M. Inoue, "Development and application of the DPLEX shield method: results of experiments using shield and segment models and application of the method in tunnel construction," Tunnelling and Underground Space Technology, vol. 11, no. 1, pp. 45-50, 1996.

[28] T. Sonoda, H. Hagiwara, H. Osaki, T. Noguchi, and M. Nakamura, "Construction of underground space by a new shield tunnelling method: spiral tunnelling and ramification of multi-circular face shield," Tunnelling and Underground Space Technology, vol. 7, no. 4, pp. 355-361, 1992.

[29] T. L. Sun, "The research status and engineering application of non-circular shield method," Journal of Railway Science and Engineering, vol. 9, pp. 1959-1966, 2017, in Chinese.

[30] H. N. Wang, G. S. Zeng, and M. J. Jiang, "Analytical stress and displacement around non-circular tunnels in semi-infinite ground," Applied Mathematical Modelling, vol. 63, pp. 303328, 2018.

[31] I. S. Sokolnikoff, Mathematical Theory of Elasticity, McGrawHill, Pennnsylvania, NW, USA, 1956.

[32] A. Z. Lu, N. Zhang, and L. Kuang, "Analytic solutions of stress and displacement for a non-circular tunnel at great depth including support delay," International Journal of Rock Mechanics and Mining Sciences, vol. 70, no. 9, pp. 69-81, 2014.

[33] A. Flamant, "Sur la répartition des pressions dans un solide rectangulaire chargé transversalement," ComptesRendus Academiedes Scincies Paris, vol. 114, pp. 1465-1468, 1892.

[34] N. I. Muskhelishvili, Some Basic Problems of the Mathematical Theory of Elasticity, Popko Noordhoff, Groningen, Netherlands, 1963.

[35] C. Q. Ru, "Eshelby inclusion of arbitrary shape in an anisotropic plane or half-plActa," Acta Mechanica, vol. 160, no. 3, pp. 219-234, 2003.

[36] W. Zou, Q. He, M. Huang, and Q. Zheng, "Eshelby's problem of non-elliptical inclusions," Journal of the Mechanics and Physics of Solids, vol. 58, no. 3, pp. 346-372, 2010.

[37] S. R. J. Heller, J. S. Brock, and R. Bart, "The stresses around a rectangular opening with rounded corners in a uniformly loaded plate," in Proceedings of the Third U.S. National Congress of Applied Mechanics, pp. 357-368, Brown University, Rhode Island, NW, USA, June 1959.

[38] F. Yang, J. Zhang, J. Yang et al., "Stability analysis of unlined elliptical tunnel using finite element upper-bound method with rigid translatory moving elements," Tunnelling and Underground Space Technology, vol. 50, pp. 13-22, 2015.

[39] E. Melan, "Der Spannungszustand der durch eine Einzelkraft im Innern beanspruchten Halbscheibe," ZAMM-Journal of Applied Mathematics and Mechanics/Zeitschrift für Angewandte Mathematik und Mechanik, vol. 12, no. 6, pp. 343346, 1932. 